\title{
Non-cell Corynebacterium parvum generated by nanotechnology: A promising immunomodulator with less side effects
}

\author{
Shangxian Gao ${ }^{\mathrm{a}, 1}$, Chenghu Liu ${ }^{\mathrm{b}, 1}$, Shoufang Qu ${ }^{\mathrm{b}}$, Jing Song ${ }^{\mathrm{b}}$, Jianfeng $\mathrm{Li}^{\mathrm{c}}$, \\ Pin Zhang ${ }^{\mathrm{a}}$, Qun Wang ${ }^{\mathrm{b}}$, Chun Guo ${ }^{\mathrm{b}}$, Fei Gao ${ }^{\mathrm{b}}$, Lining Zhang ${ }^{\mathrm{b}, *}$ \\ a Department of Medium, National Institute for the Control of Pharmaceutical and Biological Products, Beijing, PR China \\ b Institute of Immunology, Medical School of Shandong University, 44\# Wenhua Xi Road, Jinan Shandong, 250012, PR China \\ c Central Laboratory, Shandong Provincial Hospital, Jinan 250021, Shandong, PR China
}

Received 29 March 2007; received in revised form 16 May 2007; accepted 24 May 2007

\begin{abstract}
Corynebacterium parvum (CP), a kind of immunomodulator, has been well documented in immunotherapy to tumor. However, severe side effects, such as intrahepatic granulomas and scleromes in injected areas, restrict its clinical application. To minimize side effects of CP, a non-cell Corynebacterium parvum product (NCPP) was prepared by disposing CP with Nanotechnology. In present study, we compared effect of NCPP with that of CP and found: (1) NCPP with non-formaldehyde residue was easy to be absorbed without swelling and sclerome in local injected areas; (2) NCPP caused no obvious liver injury in murine and macaques; (3) NCPP maintained powerful anti-tumor activity, increased splenic index, elevated macrophage number, phagocytosis and production of hydrogen peroxide $\left(\mathrm{H}_{2} \mathrm{O}_{2}\right)$, and nitric oxide (NO); (4) Importantly, unparallel CP, NCPP could stimulate macrophages to produce low level of tumor necrosis factor- $\alpha(\mathrm{TNF}-\alpha)$ but high level of interferon- $\gamma$ (IFN- $\gamma$ ), an inhibitor to fibrosis. Our study has led to the view that NCPP will evolve into a new valuable immunomodulator for clinical application.

(C) 2007 Elsevier B.V. All rights reserved.
\end{abstract}

Keywords: Corynebacterium parvum; Tumor; Immunomodulator; Macrophage

\section{Introduction}

Corynebacterium parvum (CP), a gram-positive, anaerobic bacillus, which can be isolated from the bone marrow of healthy individuals, is not obviously pathogenic to the human body. Similar to the Bacillus

\footnotetext{
* Corresponding author. Tel.: +86 531 88380228; fax: +86 531 88382038 .

E-mail addresses: immuno@sdu.edu.cn, zhang_lining@hotmail.com (L. Zhang).

1 These authors contributed equally to this work.
}

Calmette-Guerin vaccine (BCG), which has been used as an immune adjuvant, $\mathrm{CP}$ is also a powerful nonspecific immune stimulator [1-6]. It has been observed that $\mathrm{CP}$ can elevate immune functions via the activation of macrophages [7], as well as the regulation of $\mathrm{T}$ cell immune responses [8]. In addition, $\mathrm{CP}$ is also able to promote haematopoietic stem cells to differentiate into monocytes, which in turn activates body immune system to exert its anti-cancer immune responses [9]. Although the precise mechanisms underlying $\mathrm{CP}$-elicited macrophage activation still remain obscure [10], nowadays, $\mathrm{CP}$ is considered as 
one of the best agents able to activate macrophages. Importantly, available data suggest that the administration of heat-killed $\mathrm{CP}$ in mice can induce multiple immunomodulatory effects and especially, the inhibition of tumor progression [11-13]. Unfortunately, recent investigations have demonstrated that $\mathrm{CP}$-treated mice could develop splenic intumescences and intrahepatic granulomas $[14,15]$. Such severe side effects of CP, no doubt, have extremely restricted its clinical applications. In this regard, strategies that minimize the adverse effects of CP while preserving its immunomodulatory functions have generated considerable interest. In fact, evidence has shown that cellular extraction from $\mathrm{CP}$ is also able to stimulate macrophage activation, prevent microbe infection and inhibit the lethal effect of sarcoma 180 [16], indicating that reconstructing CP while retaining its biological function is feasible. This has led us to prepare a neotype of an immunomodulator originated from CP using advanced nanotechnology.

Nanotechnology has gradually been introduced into multiple fields $[17,18]$. For instance, nanometer medicaments, the nano-size granules, always have the unique properties of being easily absorbed while preserving low concentrations and immunogenicity. In addition, the diversification of particle size will also exert effects on serum lifetime and the pattern of deposition in the primed objects. All these specialties prove that nanotechnology is a promising strategy for drug discovery and clinical application $[19,20]$.

To date, no information has been available on the transformation of CP using Nanotechnology. Therefore, the present study was designed initially to dispose of CP, using Nanotechnology to prepare a neotype of immunomodulator, referred to as the Non-cell Corynebacterium parvum Product (NCPP), and then, to investigate the general pharmacological traits of NCPP, with a specific focus on the effect of NCPP on mononuclear phagocytes.

\section{Materials and methods}

\subsection{Animals}

Male C57BL/6 and BALB/c mice (6-8 weeks, 20-22 g) were obtained from the Shanghai Laboratory Animal Center, Chinese Science Academy (Shanghai, China). Macaques (34 years, 3-5 kg) and rabbits $(2-3$ years, 3-5 kg) were purchased from the Laboratory Animal Center, Chinese Military and Medical Academy of Sciences (Beijing, China). All animals were bred under specific pathogen-free conditions. We give assurance of humane practice in animal maintenance and experimentation and all animal care and experimental treatments were done with the approval of the Shandong University Ethics Committee.

\subsection{Cell lines and reagents}

The B16 melanoma cell line was purchased from the Chinese Military and Medical Academy of Sciences (Beijing, China). CP (77-1 strain) was obtained from the National Institute for the Control of Pharmaceutical \& Biological products( Beijing, China). PE-conjugated anti-F4/80 and PEconjugated anti-IgG antibodies were purchased from eBioscience (San Diego, USA).

\subsection{Preparation of $N C P P$}

The cultured CPs were collected, sterilized and then cracked into small particles in an extra-high voltage jet crushing machine (Nanonizer, Japan). These particles were precipitated by centrifugation and resuspended in $0.9 \%$ sodium chloride solution, named NCPP, and stored at $4{ }^{\circ} \mathrm{C}$ for use. The size of NCPP, as well as CP, was detected under the transmission electron microscope (Phillips Co. Germany).

\subsection{Treatment}

B16 melanoma cells were cultured in RPMI1640 medium (Gibico) supplemented with 10\% fetal bovine serum (SiJi Qing, Hangzhou, China). For establishment of a tumor-bearing murine model, tumor cells $\left(1 \times 10^{6} / 0.1 \mathrm{ml}\right)$ were inoculated subcutaneously in the left flank of each mouse. When the maximal diameter and the minimal diameter of tumors reached about $5 \times 5 \mathrm{~mm}$, mice were injected intraperitoneally with NCPP or CP $(0.5 \mathrm{mg} / \mathrm{mouse})$ or phosphate-buffered saline (PBS) as a control. Groups were described as B16/NCPP, B16/CP, B16/PBS and PBS, respectively. In the splenic activation test, mice were sacrificed at day 14 after mice were injected intraperitoneally with NCPP or CP $(0.5 \mathrm{mg} /$ mouse $)$ and splenic index was calculated by the following formula: Splenic index $=[$ splenic weight (mg) of control group/body weight (g) of control group]/ [splenic weight (mg) of treated groups/body weight ( $\mathrm{g}$ ) of treated groups]. In the cutaneous absorbability experiment, NCPP or CP $(0.5 \mathrm{mg} / \mathrm{rabbit})$ was cutaneously injected into a rabbit's back (4 rabbits/group), and local swelling and sclerome were evaluated at $24 \mathrm{~h}, 48 \mathrm{~h}$ and $72 \mathrm{~h}$ after injection.

\subsection{Evaluation for histopathology}

Briefly, NCPP or CP (3 mg/macaque) was injected intramuscularly into macaques twice a week for 90 days and macaques were sacrificed within $24 \mathrm{~h}$ after the last injection. Similarly, NCPP or CP $(0.5 \mathrm{mg} / \mathrm{mouse})$ was injected intraperitoneally into $\mathrm{BALB} / \mathrm{c}$ mice and 10 days latter, all treated mice were sacrificed to detect liver histopathological alteration. In succession, liver tissues of macaques and mice were fixed in $4 \%$ formaldehyde $(40 \%$ formalin $100 \mathrm{ml}$, $\mathrm{Na}_{2} \mathrm{HPO}_{4} 6.5 \mathrm{~g}, \mathrm{NaH}_{2} \mathrm{PO}_{4} 4.0 \mathrm{~g}$, DDW $900 \mathrm{ml}$ ) for $24 \mathrm{~h}$ and embedded in paraffin. The sections were prepared, deparaffinized and stained with hematoxylineosin and eosin. In the end, pathologic changes were observed under a light 

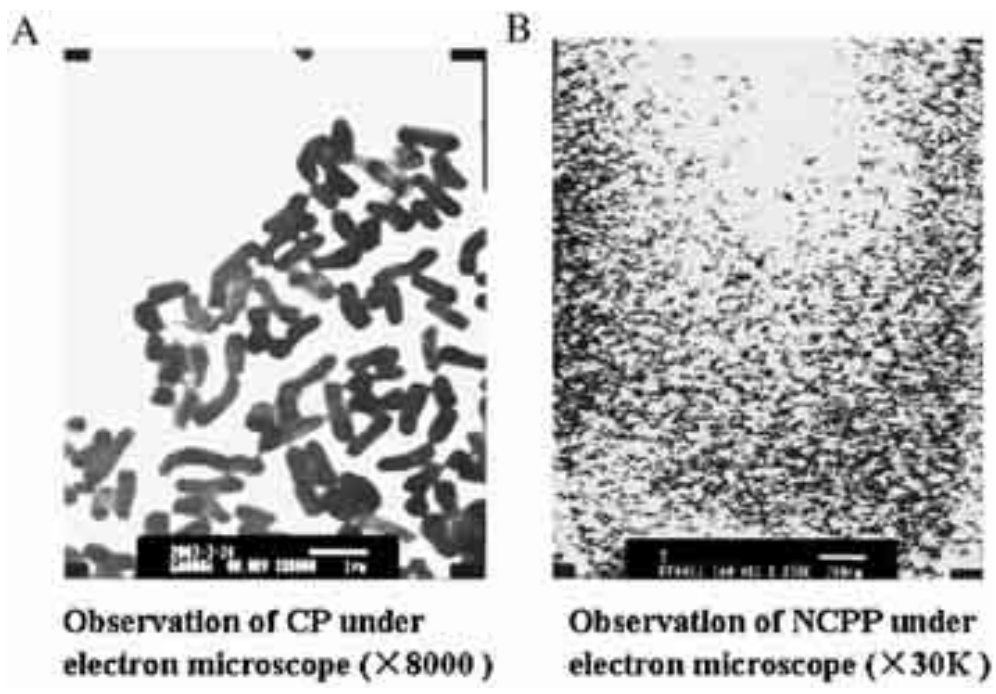

Fig. 1. Observation under electron microscope. The CP (A) and NCPP (B) which was obtained by disposing Corynebacterium parvum in extra-high voltage jet crushing machine were observed under the transmission electron microscope.

microscope and grades were evaluated according to the following standard: 0 grade: normal liver tissue; 1 grade: slight inflammatory cell infiltration and spotty necroses; 2 grade: inflammatory cell infiltration and local necroses; 3 grade: comprehensive inflammatory cell infiltration in portal area and hepatic lobule accompanying comprehensive hepatic necroses.

\subsection{The isolation and culture of macrophage}

The celiac fluid containing macrophages was collected from mice at day 10 after the injection of NCPP, CP or PBS, and then cultured in dishes for $2 \mathrm{~h}$. The attached macrophages were collected to evaluate the purity of celiac macrophage population by flow cytometry and then cultured the remanent cells in 96 well plates $\left(5 \times 10^{5} /\right.$ well $)$ in the presence of lipopolysaccharides (LPS, $10 \mathrm{ug} / \mathrm{ml}$ ) at $37^{\circ} \mathrm{C}$ for $48 \mathrm{~h}$. Finally, the supernatant was collected and used for the detection of cytokines, $\mathrm{NO}$ and $\mathrm{H}^{2} \mathrm{O}^{2}$.

\subsection{Determination of cytokines, $\mathrm{NO}$ and $\mathrm{H}_{2} \mathrm{O}_{2}$}

IFN- $\gamma$, TNF- $\alpha$ were detected by Enzyme-linked immunosorbent assay kits (BD Biosciences, USA) according to the instructions of the Cytokine ELISA Protocol. The level of NO and $\mathrm{H}_{2} \mathrm{O}_{2}$ was analyzed by a commercially available Kit (Beyotime Institute of Biotechnology, China) and measured the absorbance at $540 \mathrm{~nm}$ using a microplate reader (BioradBenchmark, USA).

\subsection{Assay for phagocytic activity of macrophages}

NCPP and CP were injected intraperitoneally into BALB/c mice. After 10 days, $1 \mathrm{ml}$ of chicken red blood cell solution

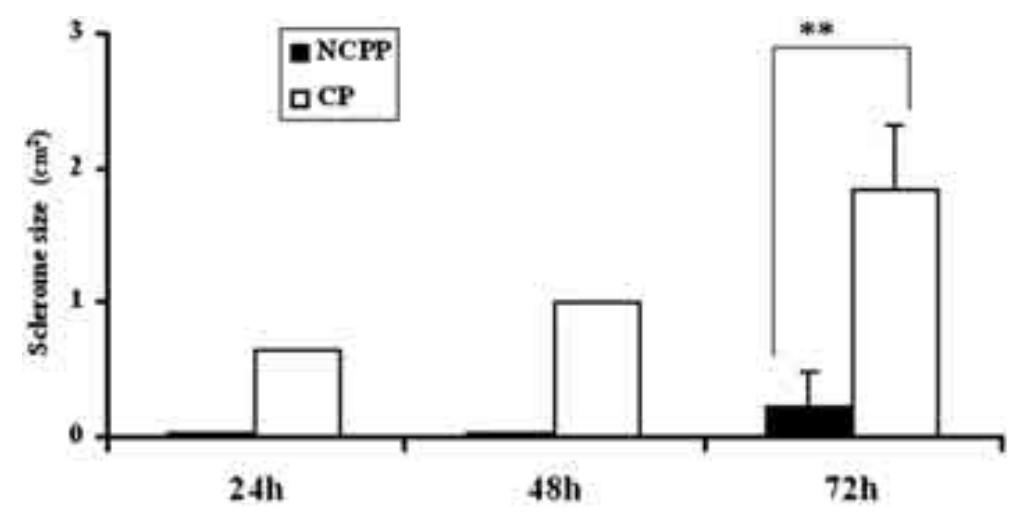

Fig. 2. The local inflammatory reaction in areas of NCPP or CP injection. NCPP or CP $(0.5 \mathrm{mg} /$ rabbit $)$ was injected cutaneously into rabbit's back. Local swelling and sclerome were evaluated at $24 \mathrm{~h}, 48 \mathrm{~h}$ and $72 \mathrm{~h}$ after treatment. ${ }^{* *} p<0.01$, compared with CP groups $(n=4)$. 

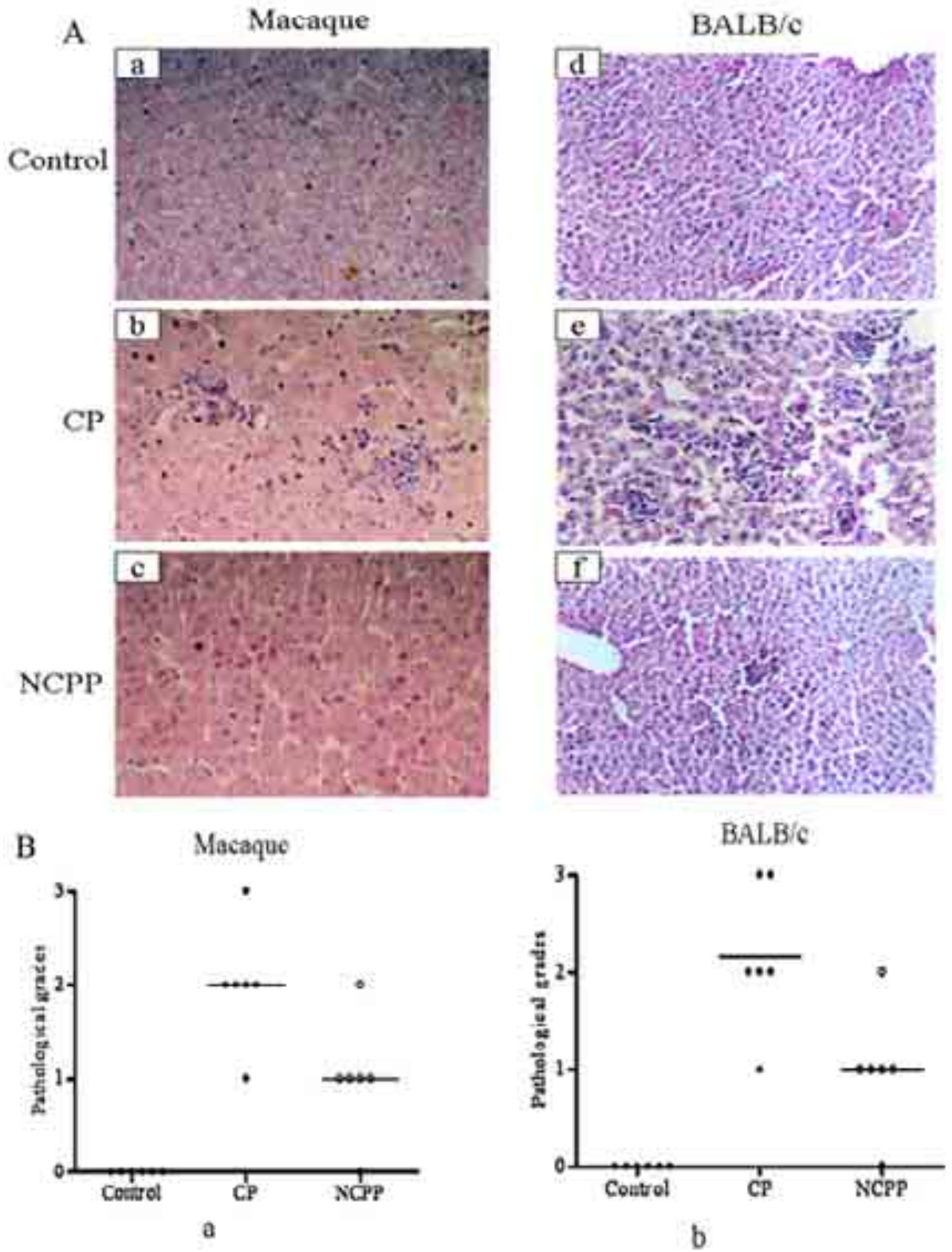

Fig. 3. The change of liver histopathology in macaques or mice after NCPP or CP treatment. A. liver histopathology of macaques and BALB/c mice: NCPP or CP was injected intramuscularly into macaques $(n=6)$ twice a week for 90 days and histopathology of liver was determined at $24 \mathrm{~h}$ after the last injection, compared with PBS control. a: PBS control ( $0.1 \mathrm{ml} / \mathrm{macaque})$; b: CP treatment (3 mg/macaque in $0.1 \mathrm{ml})$; c: NCPP treatment (3 mg/ macaque in $0.1 \mathrm{ml}) ; 0.1 \mathrm{ml}$ of PBS (d) or $0.5 \mathrm{mg} \mathrm{CP}(\mathrm{e})$ or $\mathrm{NCPP}(\mathrm{f})$ in $0.1 \mathrm{ml}$ of PBS was injected intraperitoneally into mice $(n=12)$ and 10 days later, liver histopathology was evaluated under the microscopy by staining with H-E. B. Pathologic grades were evaluated according to liver histopathologic alteration: a: macaques; b: BALB/c mice.

was given intraperitoneally. Thirty minutes later, the mice were sacrificed. The celiac fluid containing macrophages was collected, centrifuged and washed twice with PBS. After staining with Wright's solution, phagocytosis of macrophages was observed under a light microscope, and the phagocytic rate and phagocytic index were calculated.

\subsection{Flow cytometry}

$1 \times 10^{6}$ cells from bone marrow were washed twice with pyrogen-free PBS and then incubated with PE-conjugated antiF4/80 antibody or PE-conjugated anti-IgG antibody in the dark at $4{ }^{\circ} \mathrm{C}$ for $30 \mathrm{~min}$. After washing twice with PBS, cells were 
analyzed by a Cytomics ${ }^{\mathrm{TM}}$ FC 500 instrument (Beckman Coulter, USA).

\subsection{Statistical analysis}

GraphPad Prism 4 Demo Software and Student's $t$-test were used to analyze the statistical difference between groups. The $p$ value $<0.05$ was considered to be a significant difference.

\section{Results}

\subsection{Preparation of NCPP by nanotechnology}

To decrease the side effects of $\mathrm{CP}$, we prepared a non-cell Corynebacterium parvum Product (NCPP) by disposing $C$. parvum in an extra-high voltage jet crushing machine (Nanonizer, Japan). The sizes of NCPP and CP were observed under a transmission electron microscope. As shown in Fig. 1, $\mathrm{CP}(\mathrm{A})$ had an anomalistic stick configuration with $1-3 \mu \mathrm{m}$ in length, whereas NCPP (B) was composed of small, uniform nano-scale sized particles. In addition, no formaldehyde was applied during the process of NCPP.

\subsection{NCPP caused no obvious scleromes in the local injected areas}

One of the side effects of $\mathrm{CP}$ is that it can cause scleromes at the site of injection. To determine the local reaction, NCPP and $\mathrm{CP}$ were cutaneously injected into a rabbit's back, and local reactions were evaluated at $24 \mathrm{~h}, 48 \mathrm{~h}$ and $72 \mathrm{~h}$ after injection. Results showed that NCPP administration caused no obvious scleromes at the administered sites, compared with $\mathrm{CP}$ (see Fig. 2).

\subsection{NCPP possessed lower liver injury than $C P$}

It has been known that $\mathrm{CP}$ may induce intrahepatic granulomas in the treated mice [11]. In the present study, we examined the influence of NCPP on liver histopathology and serum transaminase activity in both macaque and murine models. In macaque models, NCPP or CP was injected intramuscularly into the macaque twice a week for 90 days and the histopathology of the liver was determined at $24 \mathrm{~h}$ after the last injection. Results showed that CP treatment induced comprehensive fatty degeneration and inflammatory cell infiltration, whereas injection of NCPP did not induce significant pathological damage (see Fig. 3A). The parallel histopathology alteration has also been identified in the $\mathrm{BALB} / \mathrm{c}$ mice at day 10 after NCPP or CP injection. As shown in Fig. 3A, there were many necrotic hepatocytes containing acidophilic bodies accompanying massive infiltrated inflammatory cells in the CP groups. However, there were only spotted inflammatory cells without necrotic hepatocytes in the NCPP group. Evaluation of the pathologic grade showed that pathologic lesions in the NCPP treatment group were significantly lower than that of the CP group both in BALB/c mice $(p=0.0157)$ and in macaques $(p=0.0209)$ (see Fig. 3B).
In addition, we examined ALT/AST alteration in the serum of BALB/c treated mice. Results showed that ALT/AST serum levels had no obvious increase after injection of NCPP for $24 \mathrm{~h}, 48 \mathrm{~h}$ and $72 \mathrm{~h}$ (data not shown).

\subsection{NCPP maintained strong anti-tumor and splenic activation effects of $C P$}

To identify the immunoregulatory function of NCPP, we investigated anti-tumor effects of NCPP in the tumor-bearing mice. As shown in Fig. 4A, the tumor growth in B16/NCPP and $\mathrm{B} 16 / \mathrm{CP}$ mice went slower than that of the $\mathrm{B} 16 / \mathrm{PBS}$ mice from day 11 after NCPP or CP treatment $(p<0.01)$, whereas there was no significant difference between the NCPP and CP groups. The splenic index assay showed that both NCPP and CP treatments had a significant splenic activation effect
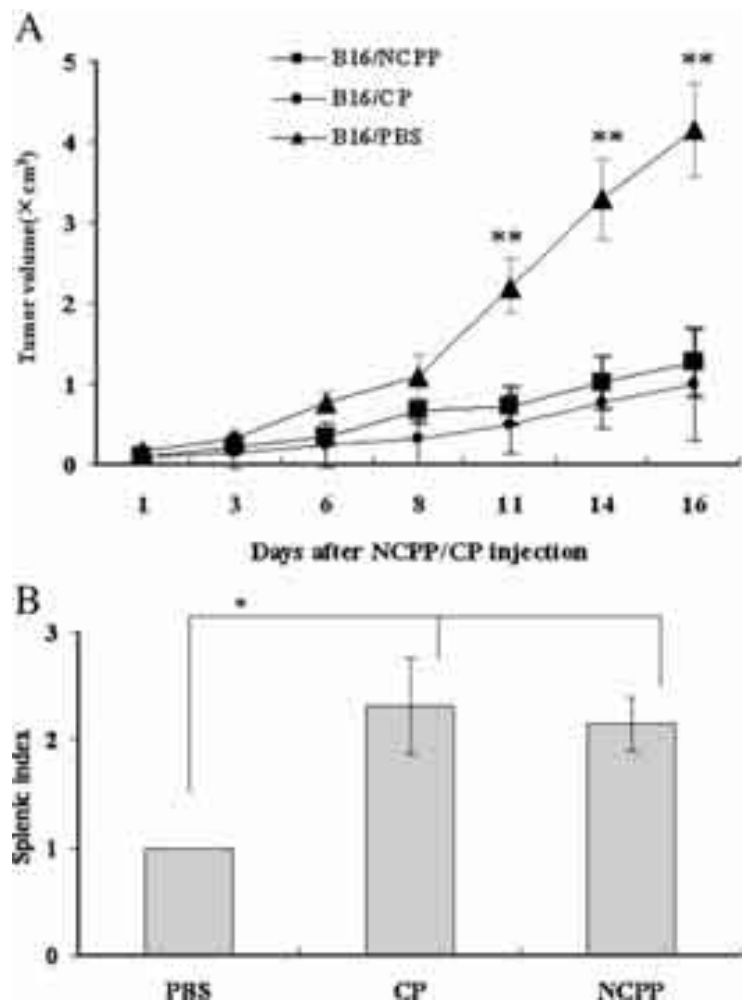

Fig. 4. Anti-tumor and splenic activation effect of NCPP. (A) B16 melanoma cells $\left(1 \times 10^{6} / \mathrm{ml}\right)$ were inoculated subcutaneously in the left flank of each mouse. When the maximal diameter and the minimal diameter of tumors arrived to about $5 \times 5 \mathrm{~mm}$, mice $(n=12)$ were injected intraperitoneally with NCPP or CP $(0.5 \mathrm{mg} / \mathrm{mouse})$ or PBS. The tumor volumes were measured three times a week. ${ }^{* *} p<0.01$, compared with B16/PBS group. (B) Mice $(n=12)$ were sacrificed at day 14 after treatment and splenic index was calculated by the following formula: Splenic index $=[$ splenic weight $(\mathrm{mg})$ of control group/body weight $(\mathrm{g})$ of control group]/[ splenic weight (mg) of treated groups/ body weight (g) of treated groups]. ${ }^{*} p<0.05$, compared with PBS control. 

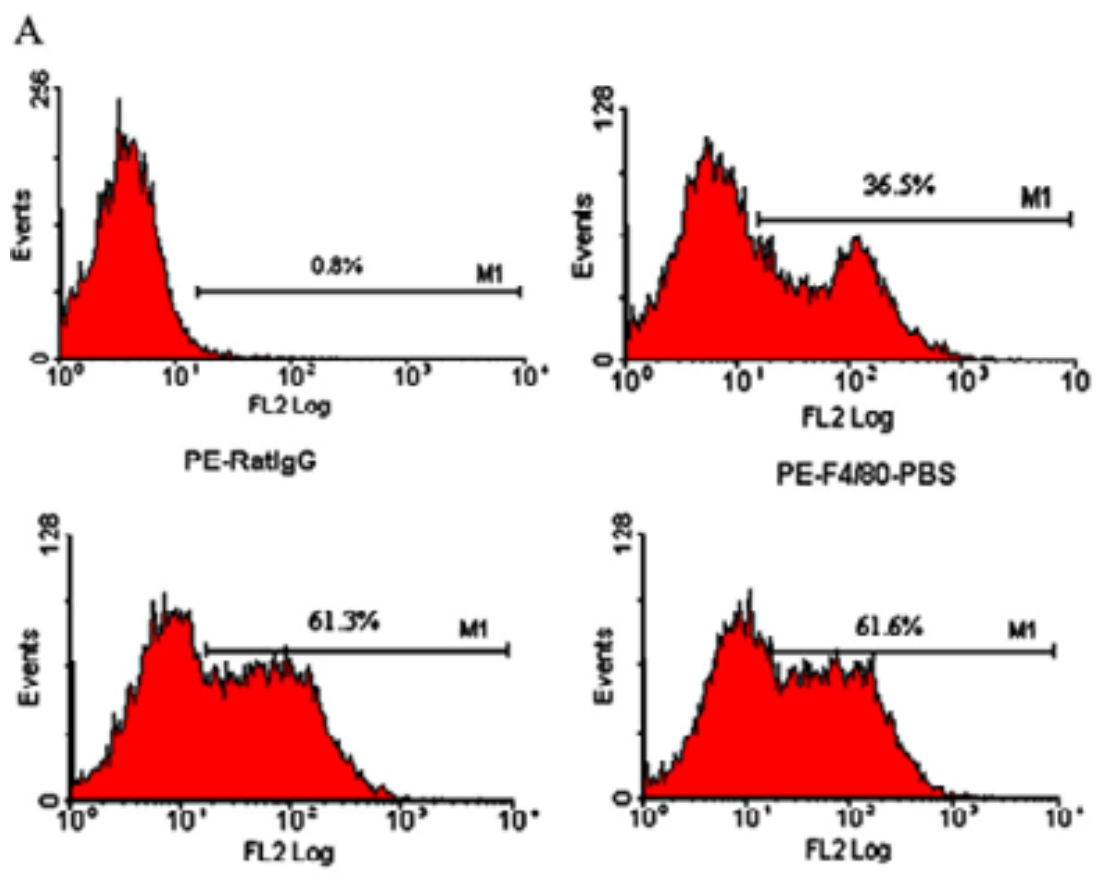

PE-F4/90-CP

PE-F4/80-NCPP

B

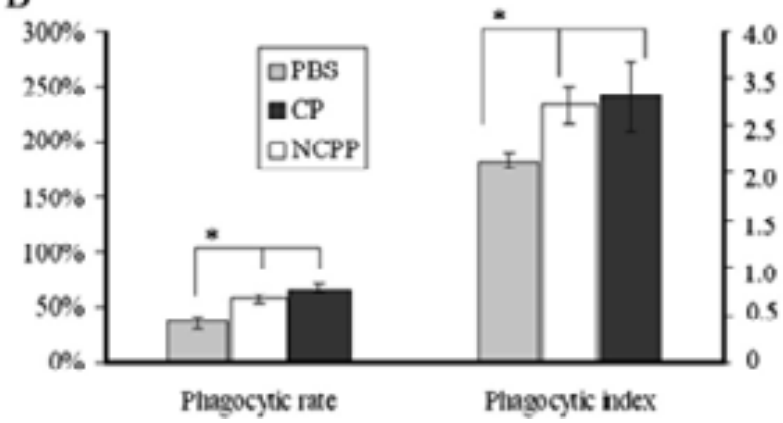

Fig. 5. Macrophage numbers and their phagocytic capacity. The cell suspension was prepared from bone marrow cells of mice treated by NCPP or CP for 10 days and stained by anti-F4/80 antibody and anti-IgG antibody. (A) The number of macrophages was detected by Flow Cytometry. (B) In phagocytic experiments of macrophages, celiac macrophages were prepared at day 10 after NCPP or CP $(0.5 \mathrm{mg} / \mathrm{mouse})$ treatment. Phagocytic rate and phagocytic index were evaluated according to the experimental instruction. ${ }^{*} p<0.05(n=12)$.

(Fig. 4B). This result demonstrated that NCPP maintained strong anti-tumor and splenic activation effects of $\mathrm{CP}$.

\subsection{NCPP could augment both macrophage number and their phagocytic capacity}

It has been reported that $\mathrm{CP}$ can enhance immune functions via the activation of macrophages. To study effect of NCPP on macrophages, we examined macrophage number, phagocytic rate and phagocytic index at day 10 after NCPP or CP treatment. Results showed that both CP and NCPP could significantly increase the number of macrophages in the bone marrow, compared with the control group $(p<0.05)$ (see Fig. 5A). The ratio of $\mathrm{F} 4 / 80^{+}$macrophages in bone marrow cells increased from $30.05 \pm 9.12 \%$ in the control group to $55.85 \pm 7.71 \%$ in the
CP group or to $59.45 \pm 3.04 \%$ in the NCPP group. At the same time, the purity of celiac macrophage population as measured by flow cytometry was $92.7 \% \mathrm{~F} 4 / 80+$ with $1.2 \% \mathrm{CD} 3+$ and $2 \%$ DX5+ indicating that we successfully obtained the purified macrophages. Moreover, NCPP as well as CP could enhance the phagocytic rate and phagocytic index of celiac macrophages, compared with the control (Fig. 5B, $p<0.05$ ).

\subsection{NCPP can enhance $\mathrm{H}_{2} \mathrm{O}_{2}$ and $\mathrm{NO}$ production of macrophages}

There is growing evidence that activated macrophages may produce an enhanced level of $\mathrm{H}_{2} \mathrm{O}_{2}$ and NO. In the following test, we tried to determine the effect of NCPP on $\mathrm{H}_{2} \mathrm{O}_{2}$ and NO production of macrophages at 10 days after NCPP or CP 
treatment. As shown in Fig. 6, both $\mathrm{CP}$ and NCPP could stimulate macrophages to produce higher levels of $\mathrm{H}_{2} \mathrm{O}_{2}$ and NO (CP vs control $P<0.05$; NCPP vs control, $P<0.01$ ). Notably, the level of NO in the NCPP treatment group was higher than in the CP treatment group $(p<0.05)$.

3.7. NCPP was able to stimulate macrophages to produce a higher level of IFN- $\gamma$ but a lower level of $T N F-\alpha$, compared with $C P$

To elucidate the influence of NCPP on the cytokine production of macrophages, macrophages were collected from celiac fluid of mice treated with NCPP or CP for 10 days and cultured in the presence of LPS for another $72 \mathrm{~h}$. The level of IFN- $\gamma$ and TNF- $\alpha$ in the supernatant was detected by the ELISA method. As shown in Fig. 7, macrophages from the control group mice secreted low levels of IFN- $\gamma$ and TNF- $\alpha$, whereas macrophages from NCPP or CP-treated mice could release enhanced IFN- $\gamma$ and TNF- $\alpha$. However, macrophages from NCPP-treated mice secreted lower levels of TNF- $\alpha$ and higher levels of IFN- $\gamma$ compared with those from CP-treated mice $(p<0.05)$, suggesting that NCPP possessed some new characteristics that $\mathrm{CP}$ did not.

\section{Discussion}

It has been shown that $\mathrm{CP}$, a strong immunological stimulant, can inhibit growth of primary and metastatic tumors and especially, the progression of cancer pleural fluid [21-25]. However, the major obstacle for further application of CP is attributed to its severe side effects. Previous works has demonstrated that the biological functions of diverse kinds of agents will be influenced once their physical configurations are transformed [26]. Thus, it is of great interest to determine whether the

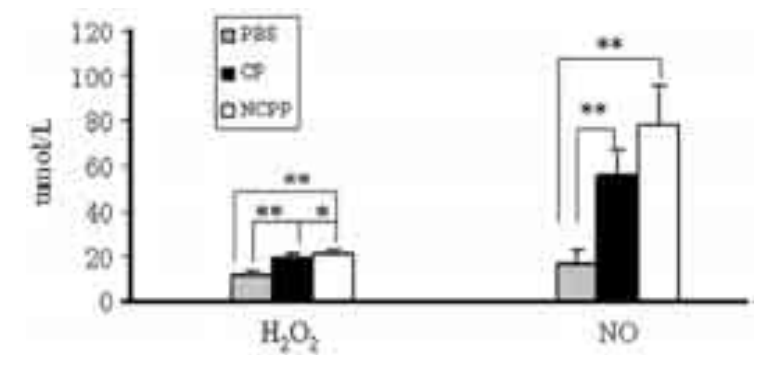

Fig. 6. The effect of NCPP on $\mathrm{H} 2 \mathrm{O} 2$ and $\mathrm{NO}$ production of macrophages. To determine effect of NCPP on $\mathrm{H}_{2} \mathrm{O}_{2}$ and NO production of macrophages, the celiac fluid containing macrophages was collected from mice at day 10 after the injection of NCPP or CP or PBS, and then cultured in dishes for 2 hours. The attached macrophages were collected and cultured in 96 -well plates $\left(5 \times 10^{5}\right.$ cells/well) in presence of $\operatorname{LPS}(10 \mathrm{ug} / \mathrm{ml})$ at $37^{\circ} \mathrm{C}$ for $48 \mathrm{~h}$. Finally, the supernatant was collected and used for detection of $\mathrm{NO}$ and $\mathrm{H}_{2} \mathrm{O}_{2}$. $* p<0.05, * * p<0.01(n=12)$.

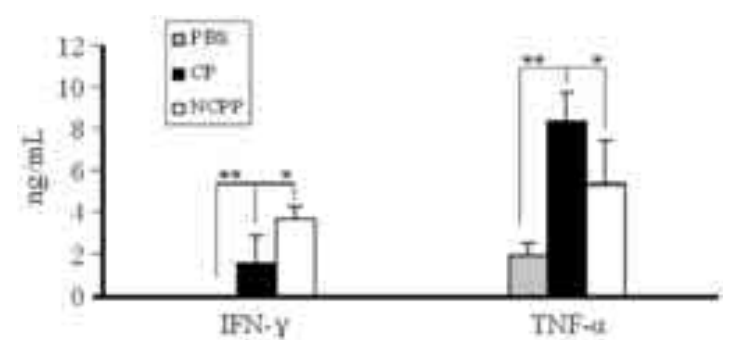

Fig. 7. The effect of NCPP on IFN- $\gamma$ and TNF- $\alpha$ production of macrophages. Briefly, the celiac fluid was collected from mice at day 10 after the injection of NCPP or CP or PBS, and then cultured in dishes for $2 \mathrm{~h}$. The attached macrophages were collected and cultured in 96 -well plates $\left(5 \times 10^{5}\right.$ cells/well $)$ in presence of LPS $(10 \mathrm{ug} / \mathrm{ml})$ at $37{ }^{\circ} \mathrm{C}$ for $48 \mathrm{~h}$. Finally, the supernatant was collected and used for detection of IFN- $\gamma$ or TNF- $\alpha$ levels with ELISA assay. $* p<0.05$, $* * p<0.01(n=12)$.

transformed CP still retains its original ability while reducing its adverse effects. In the present study, utilizing a nanometer technique, we, for the first time, tried to transform CP for the preparation of NCPP. Our results showed that NCPP did bear better general characteristics than CP (Fig. 1), which possessed small, uniform particles and contained low levels of pyrogen, suggesting that we successfully prepared a neotype of an immunomodulator. Surely, this provided the feasibility for the following experiments.

We next investigated whether NCPP possessed the biological functions pertinent to CP. In this work, we found that NCPP and CP could both significantly inhibit growth of B16 melanoma in the tumor-bearing mice compared with B16/PBS group. Moreover, other reports have shown that an increased splenic index was relevant to the anti-tumor effect of CP [27,28]. Our study demonstrated that both NCPP and CP administration were able to have significant splenic activation effects (Fig. 4). These data indicate that NCPP still maintains the immune functions similar to $\mathrm{CP}$. A recent report has revealed that $\mathrm{CP}$ is able to modulate the proliferation and differentiation activities of macrophages [7]. In this study, we presented data that both NCPP and CP can not only enhance the numbers of macrophages, but also augment the phagocytic rate and phagocytic capacity of celiac macrophages in the treated mice compared with the PBS control groups (Fig. 5); thereby confirming that NCPP retains the functions of $\mathrm{CP}$ in the activation and regulation of macrophage. It has been shown that macrophages may continuously secrete obvious levels of $\mathrm{NO}$ once activated $[29,30]$. We showed that $\mathrm{H}_{2} \mathrm{O}_{2}$ and NO levels were significantly increased in response to NCPP, indicating that NCPP is another powerful stimulator of $\mathrm{H}_{2} \mathrm{O}_{2}$ and $\mathrm{NO}$ release in macrophages 
(Fig. 6). All of the above data in this study clearly confirms that NCPP still preserves the fundamental immune functions possessed by $\mathrm{CP}$.

Now that a novel immunomodulator had been obtained, conceivably, a major concern has arisen about its safety. In this investigation, NCPP injection could not induce obvious swelling and sclerome in the treated areas (Fig. 2), suggesting that NCPP is easy to be absorbed and causes minor local inflammatory reactions. Furthermore, the present assay for histopathology in NCPP-treated macaques and mice has shown that NCPP did not induce obviously pathological injury in the livers (Fig. 3), indicating that the toxicity of NCPP has been reduced compared with $\mathrm{CP}$. In addition, we also identified the similar pathological alterations in the macaque's spleen, lung, kidney and local muscles for injection (data not shown). Our data demonstrates that, in addition to retaining the fundamental biological functions, NCPP has minimal side effects.

It has been well established that macrophages could deteriorate liver fibrosis and primary biliary cirrhosis through secreting many soluble cytokines including TGF- $\beta$, TNF- $\alpha$, and IFN- $\gamma$ [31,32]. Interestingly, TNF$\alpha$ and IFN- $\gamma$ may act with reverse roles in liver fibrosis, although they both can be secreted by activated macrophages. It has long been observed that IFN- $\gamma$ is mainly produced by NK cells and T cells. But accumulated evidence has shown that IFN- $\gamma$ can also be secreted by activated macrophages $[33,34]$.

In this work, we presented that both NCPP and CP could significantly enhance IFN- $\gamma$ and TNF- $\alpha$ secretion of macrophages (Fig. 7). Of note, a significant difference exists regarding the role of $\mathrm{NCPP}$ and $\mathrm{CP}$ in stimulating IFN- $\gamma$ and TNF- $\alpha$ secretion, i.e., NCPP could activate macrophages to secrete a higher level of IFN- $\gamma$ than CP $(p<0.05)$, whereas CP could induce higher TNF- $\alpha$ secretion than NCPP $(p<0.05)$, suggesting that NCPP may not induce fibrosis through the distinct immunoregulatory mechanism in stimulating specific cytokine secretion. Recent observations indicated that IFN- $\gamma$ was a powerful anti-fibrogenic cytokine that might play central roles in the downregulation of liver fibrosis, whereas TNF- $\alpha$ might aggravate this disease by influencing the balance of IFN- $\gamma[35,36]$. The role of NCPP from this study is, at least in part, in agreement with these conclusions.

In summary, we successfully reconstructed a neotype of immunomodulator, NCPP, through the transformation of CP utilizing nanometer technique. We confirmed that NCPP not only retains the fundamental biological functions of CP, but also possesses lower side effects, implying that NCPP may be a better immunoregulator than CP. Further studies are required to elucidate the mechanisms of how NCPP affects macrophages and other immunocytes in order to fulfill its widespread clinical applications in the future.

\section{Acknowledgments}

This work was supported by the Natural Science Foundation of China (Grant No. \#30271247, \#30371352), National 973 Project of China (Grant No. \#2006CB503803).

\section{References}

[1] Koukalova D, Kod'ousek R, Hajek V, Kolar M. Experimental nonspecific immunostimulation by the Propionibacterium acnes vaccine. J Acta Univ Palacki Olomuc Fac Med 1992;133:19-23.

[2] Kato K, Yamamoto K, Kimura T, Azuma I, Askenase PW. Suppression of BCG cell wall-induced delayed-type hypersensitivity by pretreatment with killed BCG: induction of nonspecific suppressor $\mathrm{T}$ cells by the adjuvant portion (MDP) and of specific suppressor $\mathrm{T}$ cells by the antigen portion (TAP). J Immunol 1984;132:2790-5.

[3] Purnell DM, Bartlett GL, Kreider JW, Biro TG, Kontra J. Comparative antitumor effects of Corynebacterium parvum, Bordetella pertussis, Bacillus Calmette-Guerin, and levamisole alone or in combination with cyclophosphamide in the CaD2 murine mammary adenocarcinoma system. Cancer Res 1979;39: 4838-42.

[4] Uede T, Huff TF, Ishizaka K. Formation of IgE-binding factors by rat $\mathrm{T}$ lymphocytes. V. Effect of adjuvant for the priming immunization on the nature of $\operatorname{IgE}$ binding factors formed by antigenic stimulation. J Immunol 1982;129:1384-90.

[5] El Kappany H, Chopra C, Nigam VN, Brailovsky CA, Elhilali M. The antitumour activity of maltose tetrapalmitate compared with other immunoadjuvants, and its effectiveness after tumour surgery. Br J Cancer 1980;42:703-11.

[6] Alaba O. Specific enhancement of immune responses by BCG: isolation of extracellular DNA from supernatants of specifically stimulated BCG-primed lymphoid cells. J Immunol 1980;124: 2688-92.

[7] Rossol S, Voth R, Brunner S, Muller WE, Buttner M, Gallati H, et al. Corynebacterium parvum (propionibacterium acnes): an inducer of tumor necrosis factor-alpha in human peripheral blood mononuclear cells and monocytes in vitro. Eur J Immunol 1990;20:1761-5.

[8] Thatcher N, Crowther D. Effects of BCG and Corynebacterium parvum on immune reactivity in melanoma patients. Dev Biol Stand 1977;38:449-53.

[9] Matsui K, Yoshimoto T, Tsutsui H, Hyodo Y, Hayashi N, Hiroishi K, et al. Propionibacterium acnes treatment diminishes $\mathrm{CD} 4+\mathrm{NK} 1.1+\mathrm{T}$ cells but induces type I T cells in the liver by induction of IL-12 and IL-18 production from Kupffer cells. J Immunol 1997;159: 97-106.

[10] Keller R, Keist R, Van der Meide PH, Groscurth P, Aguet M, Leist $\mathrm{TP}$, et al. Induction, maintenance, and reinduction of tumoricidal activity in bone marrow-derived mononuclear phagocytes by Corynebacterium parvum. Evidence for the involvement of a T 
cell- and interferon-gamma-independent pathway of macrophage activation. J Immunol 1987;138:2366-71.

[11] Fisher B, Wolmark N, Saffer E, Fisher ER. Inhibitory effect of prolonged Corynebacterium parvum and cyclophosphamide administration on the growth of established tumors. Cancer 1975;35:134-43.

[12] Haskill S, Ritter F, Becker S. Effect of C. parvum on intratumor immunity to the T1699 mammary adenocarcinoma. J Immunol 1980;125:454-8.

[13] Millman I, Scott AW, Halbherr T. Antitumor activity of Propionibacterium acnes (Corynebacterium parvum) and isolated cytoplasmic fractions. Cancer Res 1977;37:4150-5.

[14] Riveros-Moreno V, Niblock A. Chemical properties of the principle in C. parvum that produces splenomegaly in mice. Immunology 1979;36:495-9.

[15] Tsuji H, Mukaida N, Harada S, Kaneko S, Matsushita E, Nakanuma $\mathrm{Y}$, et al. Alleviation of lipopolysaccharide-induced acute liver injury in Propionibacterium acnes-primed IFN-gdeficient mice by a concomitant reduction of TNF-a, IL-12, and IL-18 production. J Immunol 1999;162:1049-55.

[16] Mara M, Julak J, Bednar M, Ocenaskova J, Mikova Z, Dohnalova A, et al. The influence of Propionibacterium acnes (Corynebacterium parvum) fractions on immune response in vivo. Zentralbl Bakteriol 1994;281:549-55.

[17] Chan VS. Nanomedicine: an unresolved regulatory issue. Regul Toxicol Pharmacol 2006;46:218-24.

[18] Jain KK. The role of nanobiotechnology in drug discovery. Drug Discov Today 2005;10:1435-42.

[19] Groneberg DA, Giersig M, Welte T, Pison U. Nanoparticle-based diagnosis and therapy. Curr Drug Targets 2006;7:643-8.

[20] Lin H, Datar RH. Medical applications of nanotechnology. Natl Med J India 2006;19:27-32.

[21] Squaiella CC, Ananias RZ, Mussalem JS, Braga EG, Rodrigues EG, Travassos LR, et al. In vivo and in vitro effect of killed Propionibacterium acnes and its purified soluble polysaccharide on mouse bone marrow stem cells and dendritic cell differentiation. Immunobiology 2006;211:105-16.

[22] Ananias RZ, Braga EG, Longhini ALF, Mussalern JS, Squaiella $\mathrm{CC}$, Longo-Maugeri IM, et al. In vitro non-specific anti-tumoral activity and surface molecules expression in cells obtained from mice treated with killed Propionibacterium acnes. 11th International Congress of Immunology, 54 (Suppl. 1). Scand J Immunol; 2001. p. 133

[23] Webb HE, Oaten SW, Pike CP. Treatment of malignant ascitic and pleural effusion with Corynebacterium parvum. Br Med J 1978;1:338-40.

[24] Thatcher N, Lamb B, Swindell R, Crowther D. Effects of Corynebacterium parvum on cellular immunity of cancer patients, assayed sequentially over 63 days. Cancer 1981;47:285-90.
[25] Rossi GA, Felletti R, Balbi B, Sacco O, Cosulich E, Risso A, et al. Symptomatic treatment of recurrent malignant pleural effusions with intrapleurally administered Corynebacterium parvum. Clinical response is not associated with evidence of enhancement of local cellular-mediated immunity. Am Rev Respir Dis 1987;135:885-90.

[26] Marel M, Pospisil M, Fiserova A, Melinova L, Skacel Z, Bednar M. Monitoring natural killer cell activity in the blood of patients with malignant pleural effusion after intrapleural administration of Corynebacterium parvum. Cas Lek Cesk 1990;129:498-500.

[27] Chare MJ, Baum M. The effect of Corynebacterium parvum on the proliferation of monocyte precursors in the bone marrow of mice. Dev Biol Stand 1977;38:195-200.

[28] Adlam C, Reid DE. Comparative studies on the cell wall composition of some anaerobic coryneforms of varying lymphoreticular stimulatory activity. Dev Biol Stand 1977;38:115-20.

[29] Ding AH, Nathan CF, Stuehr DJ. Release of reactive nitrogen intermediates and reactive oxygen intermediates from mouse peritoneal macrophages. Comparison of activating cytokines and evidence for independent production. J Immunol 1988;141: 2407-12.

[30] Stuehr DJ, Gross SS, Sakuma I, Levi R, Nathan CF. Activated murine macrophages secrete a metabolite of arginine with the bioactivity of endothelium-derived relaxing factor and the chemical reactivity of nitric oxide. J Exp Med 1989;169: 1011-20.

[31] Munder M, Mallo M, Eichmann K, Modolell M. Murine macrophages secrete interferon gamma upon combined stimulation with interleukin (IL)-12 and IL-18: a novel pathway of autocrine macrophage activation. J Exp Med 1998;187:2103-8.

[32] Fultz MJ, Barber SA, Dieffenbach CW, Vogel SN. Induction of IFN-gamma in macrophages by lipopolysaccharide. Int Immunol 1993;5:1383-92.

[33] Furukawa F, Matsuzaki K, Mori S, Tahashi Y, Yoshida K, Sugano Y, et al. p38 MAPK mediates fibrogenic signal through Smad3 phosphorylation in rat myofibroblasts. Hepatology 2003;38:879-89.

[34] Tsikrikoni A, Kyriakou DS, Rigopoulou EI, Alexandrakis MG, Zachou K, Passam F, et al. Markers of cell activation and apoptosis in bone marrow mononuclear cells of patients with autoimmune hepatitis type 1 and primary biliary cirrhosis. J Hepatol 2005;42:393-9.

[35] Casali A, Gionfra T, Rinaldi M, Tonachella R, Tropea F, Venturo I, et al. Treatment of malignant pleural effusions with intracavitary Corynebacterium parvum. Cancer 1988;62:806-11.

[36] Koukalova D, Kod'ousek R, Hajek V, Kolar M. Experimental nonspecific immunostimulation by the Propionibacterium acnes vaccine. Acta Univ Palacki Olomuc Fac Med 1992;133:19-23. 